\title{
Be Star Discs: Inflow or Outflow?
}

\author{
John M. Porter \\ Astrophysics Research Institute, Liverpool John Moores University, \\ Twelve Quays House, Egerton Wharf, Birkenhead L41 1LD. UK
}

\begin{abstract}
It is assumed that the dynamics of Be star discs is dominated by the effects of viscous stresses. By examining angular momentum transport in discs, we show that many, if not all observed Be star discs should be accretion discs unless (i) the disc is acted upon by another agent (e.g. magnetic fields or the stellar radiation field), or (ii) the disc cools significantly as it flows outwards.
\end{abstract}

\section{Introduction}

Theories of Be star disc formation include wind compressed discs (Bjorkman \& Cassinelli 1993), outflowing viscous discs (Lee, Saio \& Osaki 1991), and wind bistability (Lamers \& Pauldrach 1991). The major problem with wind generated discs is that the disc appears to be rotating in a Keplerian fashion (e.g. Hanuschik 1996), whereas any wind causing the equatorial enhancement is likely to conserve angular momentum, and should not be observed to be rotating faster than the star. Consequently wind bistability, and wind compression do not seem to be able to provide the observed discs.

Therefore the theory which seems to be most applicable to Be star discs is that of viscously driven outflow (Lee et al. 1991). Angular momentum is added to the inner edge of the disc increasing its angular velocity to slightly superKeplerian. The disc interacts with itself via viscosity and angular momentum is transported outwards. The angular momentum source has been suggested by Osaki (1986) to be due to non-radial pulsations dissipating in the atmosphere of the star.

The outflowing viscous disc model has several circumstantial corroborations - the variation in the line asymmetry (the $V / R$ variation) can be naturally generated in Keplerian discs (Papaloizou et al. 1992, Okazaki 1991). Excess IR emission generated by the disc is seen to disappear over a timescale of months (Hanuschik et al. 1993) which is similar to the viscous timescale in these discs this is perhaps indicative of phases of outflow and inflow within the disc.

Let us assume that Be star discs are viscous discs and confront the theory with observations. Here we ask if a standard set of assumptions regarding viscous discs can lead to the sort of discs existing around Be stars. Specifically we examine if we can detect whether the disc flows inwards (a normal accretion disc) or outwards (termed an "excretion disc" or a "decretion disc" in the literature). 


\section{The disc structure}

Excretion or decretion discs are characterised by the surface density $\Sigma=\rho(R, 0) H$, where $\rho(R, 0)$ is the density in the equatorial plane at radius $R$, and $H$ is the density scale height $H=\frac{R c_{s}}{v_{\phi}}$ where $c_{s}$ is the sound speed and $v_{\phi}$ is the rotational velocity (a cylindrical co-ordinate system $R, \phi, z$ is used throughout). Typically the discs are assumed to be isothermal in the $z$ direction (but see Millar, these proceedings). For discs dominated by viscosity, rotational velocities are nearly Keplerian (e.g. Shakura \& Sunyaev 1973) $v_{\phi} \approx \sqrt{G M_{*} / R}$, where $M_{*}$ is the mass of the star, and $G$ is the gravitational constant.

The density in the equatorial plane $\rho(R, 0)$ is assumed to be a power law

$$
\rho(R, 0)=\rho_{0}\left(\frac{R}{R_{*}}\right)^{-n}
$$

(note that the numerical simulations of viscous discs by Narita et al. 1994 have power law surface densities from $R \gtrsim 2 R_{*}$, making this assumption a good one beyond the inner parts of the disc).

\section{Angular momentum transport}

Using the assumptions above for the disc structure, the angular momentum transport may now be examined. The disc is assumed to be steady (so $\partial / \partial t=0$ ). Conservation of angular momentum in the disc yields

$$
\frac{1}{R} \frac{\partial}{\partial R}\left[R \Sigma v_{R} R^{2} \Omega-R \Sigma \nu R^{2}(\partial \Omega / \partial R)\right]=0
$$

( $\Omega$ is the angular velocity, $v_{R}$ is the radial velocity, and $\nu$ is the viscosity, taken to be the Shakura-Sunyaev 1973 value $\nu=\alpha c_{s} H$ ) which when integrated produces

$$
v_{R} \Omega-\nu \frac{\partial \Omega}{\partial R}=\frac{L}{\Sigma R^{3}}
$$

where $L$ is a constant set at the inner boundary describing the input of angular momentum to the disc.

With the Keplerian angular velocity $\Omega=\sqrt{G M_{*} / R^{3}}$, this reduces to

$$
v_{R}=-\frac{3}{2} \frac{\nu}{R}+\frac{L}{\Sigma \sqrt{G M_{*} R^{3}}}
$$

and so the radial velocity is positive (i.e. outflow) if

$$
L>\frac{3}{2} \nu \Sigma \sqrt{G M R}
$$

As $L$ is a constant, then the right hand side must not grow with radius to ensure the limit is adhered to even for large radii, and so $\nu \Sigma \sqrt{R}$ must have a constant or falling radial dependence.

Inserting the density structure from the last section, and examining powers of radial co-ordinate produces the limit that for positive radial flow (i.e. outflow) is then $3 \frac{1}{2}-n<0$ for isothermal discs. (The evidence for radial non-isothermality in discs is not strong and so we assume here that $c_{s}$ is a constant.) 


\section{Observational constraints \& conclusions}

Values of the power law index $n$ may be constrained from fitting the IR excess of Be stars in a fashion similar to that described in Waters (1986). The power law index $n$ from eq. 1 may be related to the power law index in Waters' (1986) "disc model" which is denoted as $\mathrm{N}$. The disc model has a constant opening angle and so the height of the disc $H \propto R$, whereas a viscous disc has $H \propto R^{3 / 2}$. Therefore $\mathrm{N}=n+1 / 2$.

Typically, $\mathrm{N}$ has been found to range from $2.0-3.5$ derived from fitting the IR excess. This wavelength regime corresponds to the inner $10 \mathrm{~s}$ of $R_{*}$ of the disc. Therefore values of $n$ range from 1.5-3.0.

With these values the radial dependence of the right hand side of eq. 5 is positive, and so at some radius the disc will cease to flow outward. The exact radius at which this occurs is dependent on the angular momentum input for initially small radial flows (required so that the B star does not spin-down significantly during its main sequence lifetime; Porter 1998) this radius will be close to the star.

We conclude then that for Be star discs to be extended, the observationally derived results state that Be star discs are likely to be accretion discs.

\section{Are Be star discs inflowing...?}

There are two necessary components to make an accretion disc: (i) a source of matter, and (ii) a specific angular momentum higher than Keplerian at the star's surface. The gas may come from a companion star or from re-accretion of the star's radiatively driven wind if it is diffuse enough (Porter \& Skouza 1999). Radiation driven winds from the central star conserve their angular momentum, and so when the weak winds re-accrete they should not form a disc as they have less angular momentum than necessary. Unless there is some significant angular momentum input via e.g. a magnetic field, then the re-accreted wind can not make a disc.

Therefore either (i) all Be stars have companions or (ii) all Be stars have weak winds and a dynamically important magnetic field. These requirements for Be stars appear too restrictive - why have the companions not been seen in all cases? Also, some Be stars do not have weak winds. Therefore the accretion disc hypothesis is rejected, and the assumptions leading to our conclusions are re-examined.

\section{6. .... or outflowing discs?}

In order for Be star discs to be outflowing, the radial dependence of the righthand side of eq. 5 must be negative. This may be achieved, for example, with discs having a radial temperature gradient. If the temperature is represented by a power law $T \propto R^{-m}$, then $c_{s} \propto R^{-m / 2}$. For $n=1.5$ and 3.0, discs have positive radial velocity if $m>4 / 3$ and $1 / 3$, respectively. If the disc does cool significantly, then the discs can flow outwards. Millar \& Marlborough (1998) have shown that in the inner 10's of $R_{*}$, the temperature does not show radial gradients sufficient to cause outflow. 
The dynamics of the disc may not solely be driven by viscosity, and another agent is also acting. This may be the radiation field of the star (for example via optically thin lines, Chen \& Marlborough 1994), or some aspect of magnetic fields. This can then support the disc and drive it outwards.

If we assume that the discs are outflowing, then we recognise that there are inconsistencies in the approach. We must also consider that one or several of the assumptions of the model are incorrect. We have not attempted to solve the equation of radial momentum and have assumed a Keplerian disc. If radial motions are significant, then the assumption of Keplerian rotation will be suspect.

\section{Conclusion}

We have assumed that Be star discs are driven by viscosity by assuming relevant expressions and discussing them in the framework of simple observational constraints derived from IR excess displayed by Be stars. We have asked whether the direction of motion of the gas can be detected (inflow or outflow). For the range of observationally derived disc density fields, we find that Be star discs are more likely to be inflowing i.e. the Be stars have accretion discs. Accretion discs seem inappropriate for Be stars as either all of them have mass-losing companions, or significant amounts of angular momentum must be added to the wind which also must be weak.

Outflowing discs are possible if there is a significant radial temperature gradient, or that the dynamics of the disc are affected by another agent - either the star's radiation field or magnetic fields. There is little evidence for sufficient radial temperature gradients, and so we conclude that the disc must be partly driven by radiation or magnetism.

\section{References}

Bjorkman, J.E., Cassinelli, J.P. 1993, ApJ 409, 429

Chen, H., Marlborough, J.M. 1994, ApJ 427, 1005

Hanuschik, R.W., Dachs, J., Baudzus, M., Thimm, G. 1993, A\&A 274, 356

Hanuschik, R.W. 1996, A\&A 308, 170

Lamers, H.J.G.L.M., Pauldrach, A.W.A. 1991, A\&A 244, L5

Lee, U., Saio, H., Osaki, Y. 1991 MNRAS 250, 432

Millar, C.E., Marlborough, J.M. 1998, ApJ 494, 715

Narita, S., Kiguchi, M., Hayashi, C. 1994, PASJ 46, 575

Okazaki, A.T. 1991, PASJ 43, 75

Osaki, Y. 1986, PASP 98, 30

Papaloizou, J.C., Savonije, G.J., Henrichs, H.F. 1992, A\&A 265, L45

Porter, J.M. 1998, A\&A 333, L83

Porter, J.M., Skouza, B.A. 1999, A\&A 344, 205

Shakura, N.I, Sunyaev, R.A. 1973, A\&A 24, 337

Waters, L.B.F.M. 1986, A\&A 162, 121 